\title{
Análisis de hemoglobinopatías en regiones afrocolombianas usando muestras de sangre seca de cordón umbilical
}

\author{
Analysis of hemoglobinopathies in afro-colombian \\ regions using dried blood samples of umbilical cord
}

\author{
María Jimena Rosero, Antonio José Bermúdez • Bogotá, D.C. (Colombia)
}

\section{Resumen}

Introducción: las hemoglobinopatías constituyen el grupo de trastornos genéticos más frecuentes a nivel mundial, con mayor prevalencia en población negra. En Colombia hay regiones de alta densidad poblacional de afrodescendientes, lo cual plantea la necesidad de establecer estrategias para la identificación temprana de los portadores y enfermos con propósitos de prevención.

Objetivo: realizar la identificación temprana de hemoglobinopatías en muestras de sangre seca de cordón umbilical.

Material y métodos: para las muestras de sangre seca se utilizaron tarjetas de tamizaje neonatal rutinario de hipotiroidismo congénito. La sangre es eluida por medio de soluciones buffer acuosas y luego se aplican para separación por electroforesis en geles de isoelectroenfoque y en columna de electroforesis capilar.

Resultados: de 5141 muestras recibidas de sangre seca de cordón umbilical, se descartaron 1294 por problemas de calidad, se procesaron 3847 (75\%) y de éstas 3244 (84\%) fueron útiles para el estudio. Se encontraron 288 (7\%) con alguna variante de hemoglobina, $\mathrm{FC}(1,88 \%), \mathrm{FS}(4,65 \%), \operatorname{SC}(0.06 \%), \mathrm{SS}(0,12 \%)$, Bart $(0,18 \%)$, FC/otro $(0,12 \%)$

Conclusiones: las muestras de sangre seca de cordón umbilical permiten realizar el estudio por electroforesis capilar y por isoelectroenfoque de las diferentes variantes de hemoglobinas anormales, que puede ser aplicable al diagnóstico temprano, siempre que se cumplan requisitos de calidad, en la aplicación de la muestra y en el tiempo de entrega para análisis. (Acta Med Colomb 2012: 37: 118-124)

Palabras clave: tamizaje neonatal, isoelectroenfoque, electroforesis capilar, rasgo falciforme, drepanocitosis, mutación

\footnotetext{
Abstract

Introduction: the hemoglobinopathies represent the most common group of genetic disorders worldwide, with higher prevalence in black population. In Colombia there are regions with high density population of African descent, which raises the need to develop strategies for early identification of carriers and patients with preventive purposes.

Objective: realize early identification of hemoglobinopathies in dried blood samples from the umbilical cord.

Material and methods: for the dried blood samples we used routine neonatal screening cards for congenital hypothyroidism. The blood is eluted by means of aqueous buffer solutions and then applied for separation by electrophoresis in isoelectric focusing gels and in capillary electrophoresis column.

Results: of the 5141cord dried blood samples received, 1294 were discarded due to quality problems; $3847(75 \%)$ were processed and of these, $3244(84 \%)$ were appropriate for the study. We found $288(7 \%)$ with some hemoglobin variant, $\mathrm{FC}(1.88 \%), \mathrm{FS}(4.65 \%), \mathrm{SC}(0.06 \%), \mathrm{SS}(0.12 \%)$, Bart $(0.18 \%)$, FC / other (0.12\%). (Acta Med Colomb 2012: 37:xx-xx)

Conclusions: dried blood samples of umbilical cord allow the study of the different variants of abnormal hemoglobins by capillary electrophoresis and isoelectric focusing, which may be applicable to an early diagnosis in the neonatal screening, provided that quality requirements in the sample application and in the delivery time for analysis are accomplished. (Acta Med Colomb 2012: 37: 118-124)

Key words: neonatal screening, isoelectric focusing, capillary electrophoresis, falciform trait, drepanocytosis, mutation.
}

Dra. María Jimena Rosero Lucero: Bacterióloga Especialista; Dr. Antonio Jose Bermúdez Fernández: MSc, FETP. Grupo de Genética, Subdirección de Red Nacional de Laboratorios, Instituto Nacional de Salud. Bogotá D.C., (Colombia). Correspondencia. Dr. Antonio José Bermúdez Fernández. Instituto Nacional de Salud Grupo de Genética. Bogotá D.C., (Colombia).

E-mail: abermudez@ins.gov.co Recibido: 19/XI/2011 Aceptado: 20/VIII/2012 


\section{Introducción}

Las hemoglobinopatías son enfermedades resultantes de una anormalidad genéticamente determinada en la estructura o síntesis de la molécula de hemoglobina (1), constituida por dos cadenas alfa $\alpha$ y dos cadenas beta $\beta$, ensambladas con un átomo de hierro que constituye el grupo hemo. Son el grupo de trastornos genéticos más común, de alta prevalencia en negros de África y en los grupos étnicos de la cuenca mediterránea y del sudeste de Asia (2).

La más conocida es la enfermedad de células falciformes, un grupo de desórdenes autosómicos recesivos con la producción de hemoglobina $\mathrm{S}$, que difiere de la $\mathrm{Hb} \mathrm{A}$ normal del adulto, en un cambio de timina por adenina ( $T$ / A) en el gen de la cadena $\beta$, resultando en una sustitución del aminoácido valina por ácido glutámico en la sexta posición de la proteina (3). La consecuencia estructural es fatal para la disponibilidad tisular de oxígeno, dado que afecta la funcionalidad, la forma del eritrocito (forma de hoz) y el transporte de oxígeno.

Los trastornos de células falciformes se encuentran en pacientes que tienen el genotipo SS, homocigoto para la mutación y también en los que tienen el genotipo heterocigoto para la hemoglobina $\mathrm{S}$ y otra anormalidad de la cadena $\beta$, conformando el doble heterocigoto; tal es el caso del genotipo para la $\mathrm{Hb} \mathrm{SC}$ y para la $\mathrm{Hb} \mathrm{S} / \beta$-thalassemia (3). En la HbC hay sustitución de la lisina por el ácido glutámico en la sexta posición de la cadena $\beta$. Las talasemias, se caracterizan por una disminución en la síntesis de las cadenas de globina, se clasifican de acuerdo con la cadena de globina afectada en $\beta$ talasemia o $\alpha$ talasemia (3). El heterocigoto para cualquiera de las formas, es el portador sano, asintomático, pero en condiciones ambientales o fisiológicas de demanda aumentada de oxígeno pueden presentar síntomas, aunque en menor proporción que el homocigoto o el doble heterocigoto en quienes se presenta una enfermedad grave (4). Según la Organización Mundial de la Salud, los desórdenes de la hemoglobina representan un problema de salud pública en $71 \%$ de 229 países. Cada año nacen más de 300000 niños afectados, $83 \%$ con anemia de células falciformes y $17 \%$ con talasemia. Las hemoglobinopatías, explican aproximadamente $3,4 \%$ de las muertes en niños menores de cinco años de edad (5).

Colombia es de población mestiza, pero hay regiones de alta densidad de afrodescendientes. La prevalencia de las variantes cambia según cada región, pero en todas hay portadores sanos y hay homocigotos o dobles heterocigotos. La mejor estrategia para la prevención es la detección temprana por medio de programas poblacionales de tamizaje neonatal (6). El objetivo de este estudio fue demostrar la viabilidad de utilizar la misma muestra que actualmente se usa para el tamizaje de hipotiroidismo congénito, para tamizar las hemoglobinopatías por las metodologías de uso más común. La ventaja de utilizar la muestra de sangre seca de cordón umbilical es que se tiene una muestra estable, fácil de transportar y suficiente incluso para hacer análisis moleculares a partir del ADN, pero lo más importante es que se garantiza la cobertura, se mejora la oportunidad en el diagnóstico y se reducen costos. Por lo tanto en este estudio se aborda la utilización de la muestra de sangre seca de cordón umbilical como estrategia para ampliar el tamizaje neonatal a la detección de hemoglobinopatías, sin causar cambios en la rutina de atención del recién nacido.

\section{Material y métodos}

Para demostrar que a partir de muestras de sangre seca obtenida de cordón umbilical en la sala de parto para el tamizaje neonatal, se pueden identificar hemoglobinas anormales, se diseñó un estudio con muestreo por conveniencia, con un sesgo calculado hacia la positividad. Luego fueron procesadas por electroforesis capilar o por electroforesis de isoelectroenfoque, en forma equitativa, en condiciones idénticas a las de procesamiento de muestras para tamizaje rutinario. El isoelectroenfoque es la técnica Gold Estándar, sin embargo los resultados positivos por cualquiera de las técnicas se verificaron en forma cruzada para corregir un posible sesgo de técnica analítica.

\section{Muestras}

Se utilizaron muestras de sangre seca, en papel Whattman 903, después de su uso en el tamizaje neonatal de hipotiroidismo congénito, de manera anónima absoluta, para evitar expectativas y ansiedad tanto por parte de las personas como de sus Instituciones de salud. Se solicitó ayuda a los laboratorios de salud pública departamentales donde es alta la población afrodescendiente (7), pidiéndoles el envío al INS de las muestras utilizadas para control indirecto de tamizaje. Se excluyen del análisis las muestras de mala calidad por envejecimiento, o que tuvieran más de tres meses de tomadas. La información única disponible es la de la tarjeta de toma de muestra, pues en ningún caso se tuvo contacto con el paciente o su médico tratante.

\section{Determinación de las variantes de hemoglobina}

Se utilizaron dos métodos, electroforesis de isoelectroenfoque y electroforesis capilar. En ambos métodos se realiza la separación de las variantes de la molécula de hemoglobina, aprovechando sus diferencias en la estructura y en las propiedades fisicoquímicas, como la carga eléctrica, forma molecular y peso, el $\mathrm{pH}$ y otras, que determinan diferencias en la movilidad bajo condiciones estandarizadas de electroforesis. Se utilizan patrones de referencia para cada variante conocida. La evidencia se obtiene mediante un registro en formato digital, el cual puede ser analizado con un software de aplicación específica y que permite identificar variantes nuevas. La diferencia fundamental entre las dos metodologías radica en que en la electroforesis capilar, las moléculas cargadas son separadas por su movilidad en un buffer alcalino con un $\mathrm{pH}$ específico, mientras en la técnica de isoelectroenfoque, las moléculas 
de hemoglobina migran en función de sus puntos isoeléctricos a través del gel cargado con anfolitos. La utilización de ambos métodos permitió confirmar en forma cruzada, los casos con resultado anormal. En forma breve para la electroforesis de isoelectroenfoque se corta un disco de 3.2 $\mathrm{mm}$ de la mancha de sangre y se coloca en una microplaca con la solución de elución de hemoglobina, se deposita el hemolizado del paciente en el pozo del gel previamente montado y se deja correr por una hora 45 minutos a 30 vatios y 1200 voltios, después se hace la fijación con ácido tricloroacético al $10 \%$, se lava con agua destilada y se deja secar; para la lectura se realiza el análisis densitométrico del gel (8). Para la electroforesis capilar se corta el disco de papel de $3.8 \mathrm{~mm}$ en cada pozo del segmento, se eluye en agua destilada por 24 horas en refrigeración, se coloca cada segmento de ocho muestras en el rack de análisis con la solución hemolizante, las hemoglobinas separadas en capilares de sílice son directa y específicamente detectadas a una absorbancia de $415 \mathrm{~nm}$ (9). Los resultados de la electroforesis son evaluados gráficamente contra patrones normales y sus variantes.

\section{Análisis estadístico}

Estadística descriptiva simple, por medio del aplicativo estadístico de Microsoft office excel 2007. Para comparación de proporciones se utilizó Chi cuadrado $\left(x^{2}\right)$ con significancia al $95 \%$ y para estimar los límites de confianza de los porcentajes se utilizó el paquete estadístico Open Epi mediante el Score de Wilson con significancia al nivel de $p<0.005$. Para la correlación entre series de datos se utilizó el coeficiente de correlación de Pearson, $r$ con significancia si $\mathrm{r}=80 \%$.

\section{Consideraciones éticas}

Se acudió a muestras en papel Whattman 903, en las que no se intervino de ninguna manera con el paciente, cumpliendo estrictamente con condiciones de manejo de muestras anonimizadas, de acuerdo con los principios éticos para las investigaciones médicas en seres humanos establecidos por la Asociación Médica Mundial en su Declaración de Helsinki .

\section{Resultados}

Se recibieron 5141 muestras de sangre seca de recién nacidos, de ambos sexos y la mayoría (66\%) de régimen de aseguramiento subsidiado. Las muestras provienen de seis departamentos, escogidos por tener alta probabilidad de mostrar casos con hemoglobinopatías por su ancestralidad afro (Tabla 1). Se analizó un número similar de muestras por cada técnica, 1801 (47\%) por la técnica de electroforesis capilar (Sebia, Capillarys 2®) y $2046(53 \%)$ por la técnica de isoelectroenfoque (Perkin Elmer®), entonces hay una diferencia en este aspecto. $\left(\mathrm{Chi}^{2}=27.7, p<0.000001\right)$. En total se analizaron 3847 muestras, con resultado informativo en 3244 , en las que se encontraron 228 casos con variantes de hemoglobina y 3016 con patrón electroforético normal (Tabla 2). Para la proporción de casos el estimado de punto es $7.02 \%$ (95\% I.C.:6.17\%-7.96\%).

Los patrones electroforéticos identificados fueron F/AS, F/S, F/AC, F/SC y Bart, F/C y otra variante.(Figuras 1a y 1b). Cuatro casos fueron homocigotos para hemoglobina $\mathrm{S}$ con fenotipo neonatal (F/SS), que corresponde a una frecuencia de $(1.75 \%)$. La hemoglobina $\mathrm{S}$ es la variante más frecuente (Tabla 3 ).

De las 5141 muestras se perdieron para análisis 1294, corresponde a un estimado de punto de $25.17 \%$ (95\% I.C.: 23.99\%-26.38\%), de las cuales 480 no cumplían con los criterios de calidad de la muestra de sangre seca en papel para el tamizaje neonatal y las restantes no fueron informativas por presentar problemas de elución que se explicó en todos los casos por envejecimiento de las muestras. (Tabla 4). La diferencia de variantes entre las regiones depende de su similitud o diferencia étnica, por ejemplo al comparar la frecuencia de variantes encontradas en Chocó con San Andrés encontramos que la diferencia no es significativa $\left(C h i^{2}=0.15, p=0.695\right)$, mientras que al comparar San Andrés con Putumayo sí es significativa. $\left(C h i^{2}=20.46\right.$, $p=0.000006$ ). Por su parte cuando se compara la frecuencia de hemoglobinopatías encontradas por región, con la frecuencia de autorreconocimiento como afrocolombiano, se encuentra una correlación positiva significativa $(r=0.82$, nivel de significancia: $r \geq 0.8$ ).

Tabla 1. Descripción de las muestras recibidas según género, aseguramiento y departamento de origen.

\begin{tabular}{|l|l|c|c|}
\hline & Muestras recibidas & $\mathbf{N}$ & $\mathbf{\%}$ \\
& Total & $\mathbf{5 1 4 1}$ & $\mathbf{1 0 0}$ \\
\hline \multirow{2}{*}{ Género } & Masculino & 1884 & 37 \\
& Femenino & 2009 & 39 \\
& Sin dato & 1248 & 24 \\
\hline \multirow{2}{*}{ Régimen de afiliación } & Subsidiado & 3386 & 66 \\
& Contributivo & 604 & 12 \\
& No asegurado & 267 & 5 \\
& Sin dato & 884 & 17 \\
\hline \multirow{2}{*}{ Departamento } & Putumayo & 928 & 18 \\
& Nariño & 1211 & 24 \\
& Guajira & 671 & 13 \\
& San Andrés Islas & 645 & 13 \\
& Chocó & 983 & 19 \\
& Valle del Cauca & 703 & 14 \\
\hline
\end{tabular}

Tabla 2. Distribución de muestras según método de análisis y clase de resultado

\begin{tabular}{|l|c|}
\hline Total de muestras recibidas & $5141(100 \%)$ \\
\hline Total de muestras procesadas & $3847(75 \%)$ \\
Analizadas por electroforesis capilar & $1801(47 \%)$ \\
Analizadas por isoelectroenfoque & $2046(53 \%)$ \\
Resultado adecuado para interpretar & $3244(84 \%)$ \\
Resultado no útil para interpretar & $603(16 \%)$ \\
\hline Muestras sin procesar por problemas de calidad & $1294(25 \%)$ \\
\hline
\end{tabular}


Tabla 3. Distribución de hemoglobinas anormales encontradas por departamento.

\begin{tabular}{|c|c|c|c|c|c|c|c|c|c|c|c|c|c|}
\hline & \multicolumn{2}{|c|}{ Heterocigoto Hb C } & \multicolumn{2}{|c|}{ Heterocigoto $\mathrm{Hb} \mathrm{S}$} & \multicolumn{2}{|c|}{ Heterocigoto $\mathrm{Hb}$ S/C } & \multicolumn{2}{|c|}{ Homocigoto $\mathrm{Hb} \mathrm{S}$} & \multicolumn{2}{|c|}{ Hemoglobina de Bart } & \multicolumn{2}{|c|}{ Hemoglobina C y otro } & \multirow{2}{*}{$\frac{\text { Total }}{\mathbf{N}}$} \\
\hline & $\mathbf{N}$ & $\%$ & $\mathbf{N}$ & $\%$ & $\mathbf{N}$ & $\%$ & $\mathbf{N}$ & $\%$ & $\mathbf{N}$ & $\%$ & $\mathbf{N}$ & $\%$ & \\
\hline Putumayo & 3 & 0.09 & 2 & 0.06 & - & - & - & - & - & - & - & - & - \\
\hline Nariño & 21 & 0.65 & 32 & 0.99 & - & - & - & - & - & - & - & - & - \\
\hline Guajira & 5 & 0.15 & 20 & 0.62 & - & - & 1 & 0.03 & 1 & 0.03 & - & - & - \\
\hline San Andrés & 5 & 0.15 & 40 & 1.23 & - & - & - & - & 4 & 0.12 & - & - & - \\
\hline Chocó & 17 & 0.52 & 49 & 1.51 & 1 & 0.03 & 3 & 0.09 & 1 & 0.03 & 4 & 0.12 & - \\
\hline Valle del Cauca & 10 & 0.31 & 8 & 0.25 & 1 & 0.03 & - & - & - & - & - & - & - \\
\hline Total & 61 & - & 151 & - & 2 & - & 4 & 0.06 & 6 & 0.06 & 4 & - & 228 \\
\hline Total & - & 1.88 & - & 4.65 & - & 0.06 & - & 0.12 & - & 0.18 & - & 0.12 & 7.0 \\
\hline
\end{tabular}

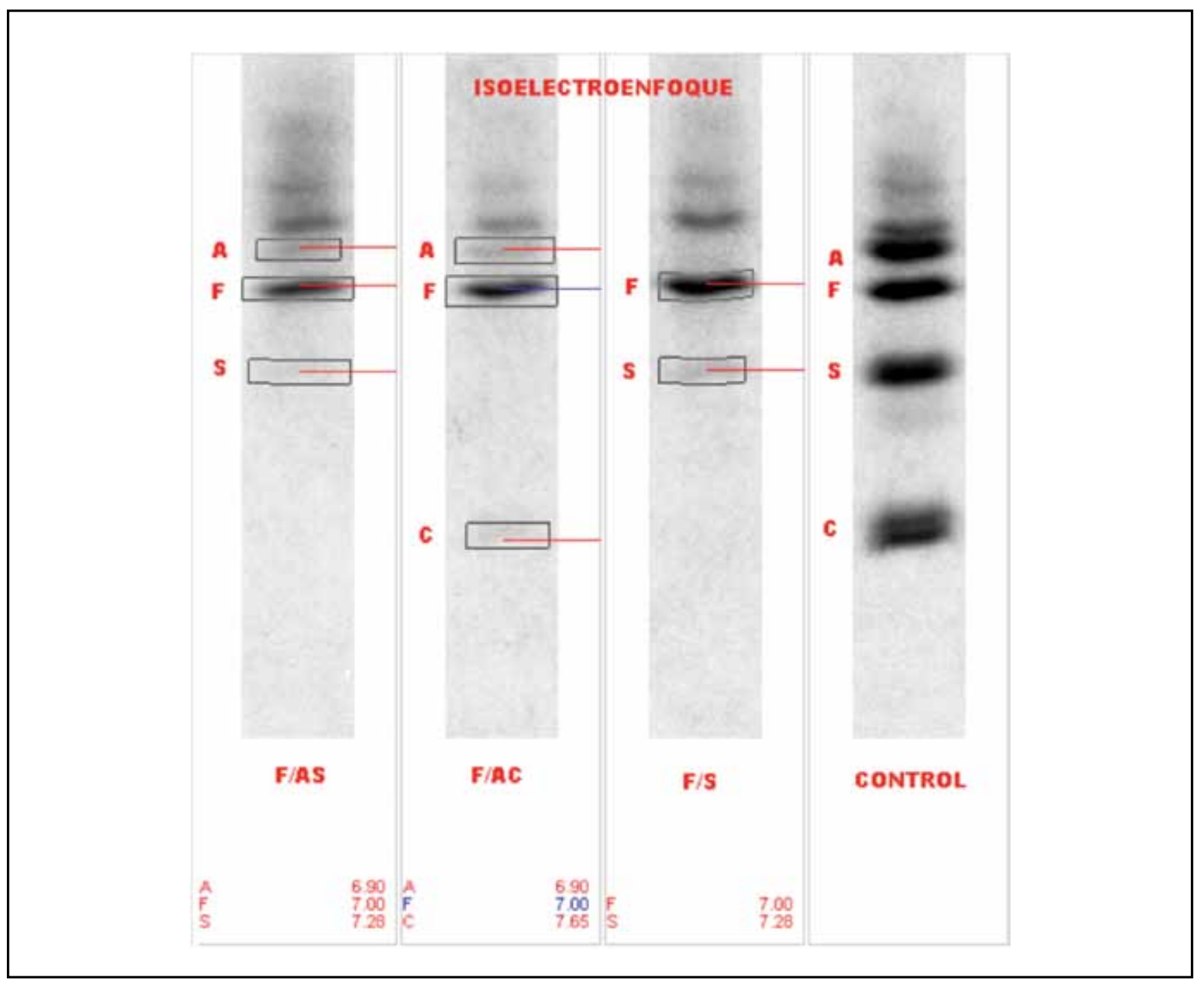

Figura 1a. Patrones electroforéticos. F/AS: heterocigoto hemoglobina S, F/AC: heterocigoto hemoglobina C, F/S homocigoto hemoglobina S y control AFSC. Muestras de sangre seca de recién nacidos analizadas por isoelectroenfoque (Perkin Elmer $\left.{ }^{\circledR}\right)$. Gel de agarosa, fuente de alimentación 30 vatios, 1200 voltios, 90 minutos. Imagen por densitometría. Scan Marker 8700. Microtek. IsoScan Imaging System. 


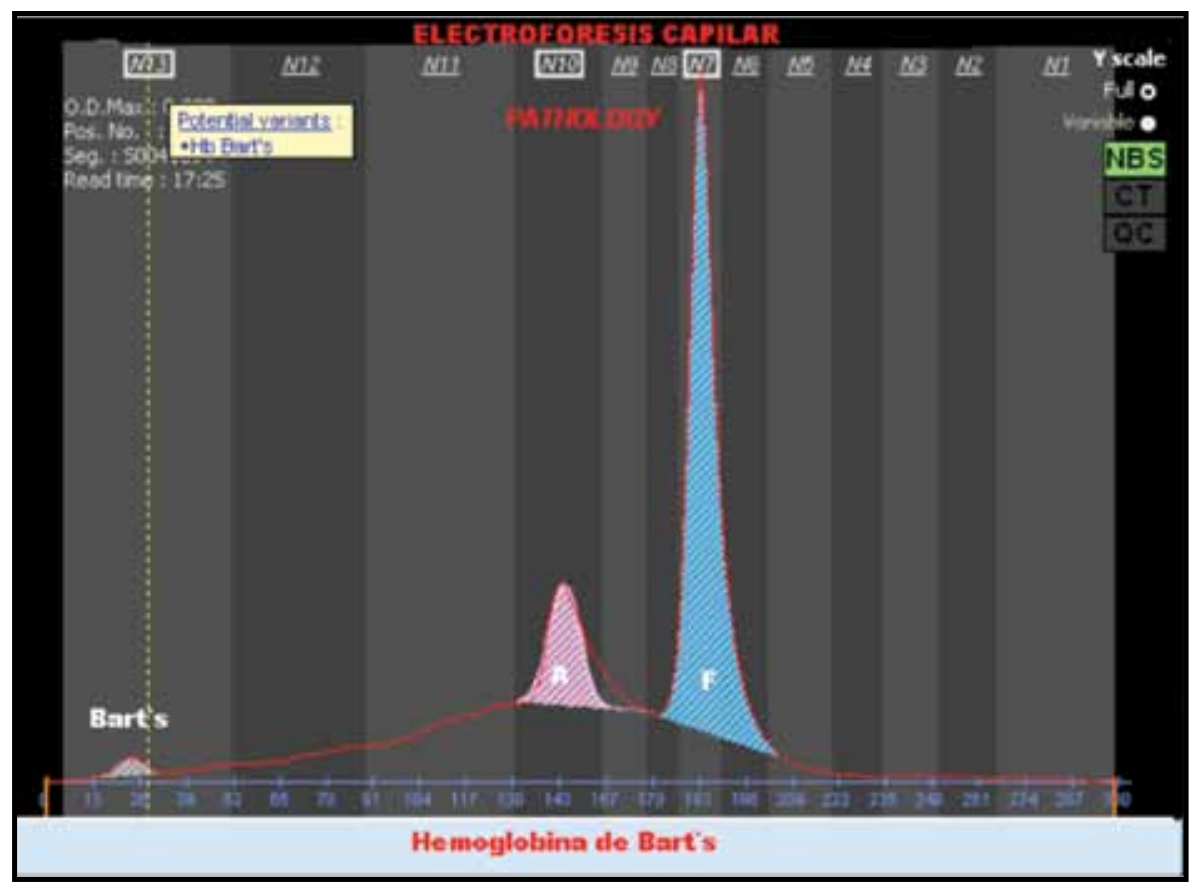

Figura 1b. Identificación de hemoglobina de Bart. Muestra de sangre seca de recién nacido analizada en el laboratorio de genética del INS, por la técnica de electroforesis capilar (Sebia ${ }^{\circledast}$ ). Muestra diluida con solución hemolizante por siete minutos, inyectada en los capilares, migración a voltaje y temperatura constante, lectura a $415 \mathrm{~nm}$. Imagen en tiempo real, por impresión de pantalla.

Tabla 4. Distribución de muestras sin procesar de acuerdo con la característica de calidad de la muestra de sangre seca.

\begin{tabular}{|l|c|c|}
\hline Calidad de la muestra & Total & Porcentaje \\
\hline Coagulada & 38 & 0.74 \\
\hline Hemolizada & 62 & 1.21 \\
\hline Coagulada-hemolizada & 3 & 0.06 \\
\hline Sobresaturada & 143 & 2.78 \\
\hline Sobresaturada-hemolizada & 16 & 0.31 \\
\hline Insuficiente & 193 & 3.75 \\
\hline Halos de suero & 16 & 0.31 \\
\hline Coagulada-sobresaturada & 7 & 0.14 \\
\hline Hemolizada-insuficiente & 2 & 0.04 \\
\hline Total & 480 & 9.3 \\
\hline
\end{tabular}

\section{Discusión}

Las muestras de sangre seca que se utilizan en el tamizaje neonatal de hipotiroidismo congénito, mostraron ser adecuadas para evaluar hemoglobinas anormales, por las técnicas de electroforesis capilar y electroforesis de isoelectroenfoque, que son las técnicas de uso actual, con la debida automatización. Otra técnica en uso es la cromatografía líquida de alta presión, HPLC, pero no se utilizó por su complejidad interpretativa (10). Los casos en los cuales la muestra no fue informativa, se debió a mala calidad de la misma, evidenciado por los criterios establecidos en forma universal para la calidad de las muestras de sangre seca en papel. Las demás muestras que no permitieron tener resultados, fueron muestras en las que se evidenció elu- ción inadecuada por tener más de tres meses de haber sido tomadas. De esta manera se evidenció que la muestra de sangre seca sirve para el propósito de tamizaje neonatal de hemoglobinopatías, siempre que se cumplan unos requisitos de calidad. También se comprobó que para el análisis sólo se utilizan pequeños discos, así queda suficiente muestra para estudios complementarios, por ejemplo los casos en los cuales se encuentran variantes nuevas según el fenotipo electroforético, los cuales requieren secuenciación del ADN para identificar la mutación causante. En este estudio todas las variantes encontradas son conocidas, por esa razón no hubo necesidad de hacer análisis del ADN, sin embargo había muestra suficiente para ese propósito.

Hubo variación en la calidad de las muestras según su origen por departamento, lo cual puede ser interpretado como diferencias en los tiempos de remisión, que tiene como consecuencia el envejecimiento de las mismas y esto genera problemas para el procesamiento (Tabla 5). Implica que para tener muestras adecuadas para análisis, se deberá dar la capacitación pertinente para explicar la importancia de la calidad en la toma de la muestra, así como la calidad en las condiciones de transporte y en la oportunidad de la entrega. La capacitación en este aspecto se evidencia como algo esencial, puesto que $25 \%$ de muestras rechazadas no es aceptable para un escenario real de tamizaje.

Aunque no era el objetivo primario del estudio, los resultados de los fenotipos encontrados son de interés, porque ratifican la alta frecuencia de las hemoglobinopatías y justifican el tamizaje neonatal. La frecuencia encontrada se relaciona con la frecuencia de auto reconocimiento como 
Tabla 5. Eficiencia en el uso de la muestra de sangre seca por departamento.

\begin{tabular}{|c|c|c|c|c|c|c|c|}
\hline \multirow[t]{2}{*}{ Departamento } & \multirow{2}{*}{$\begin{array}{c}\begin{array}{c}\text { Muestras } \\
\text { recibidas }\end{array} \\
\mathrm{N}\end{array}$} & \multicolumn{2}{|c|}{$\begin{array}{l}\text { Muestras sin procesar por } \\
\text { problemas de calidad }\end{array}$} & \multicolumn{2}{|c|}{$\begin{array}{l}\text { Resultado no adecuado } \\
\text { para interpretar }\end{array}$} & \multicolumn{2}{|c|}{$\begin{array}{l}\text { Resultado adecuado } \\
\text { para interpretar }\end{array}$} \\
\hline & & $\mathbf{N}$ & $\%$ & $\mathbf{N}$ & $\%$ & $\mathbf{N}$ & $\%$ \\
\hline Putumayo & 928 & 588 & 63.36 & 14 & 1.51 & 326 & 35.13 \\
\hline Nariño & 1211 & 186 & 15.36 & 277 & 0.50 & 748 & 61.77 \\
\hline Guajira & 671 & - & - & 10 & 1.49 & 661 & 98.51 \\
\hline San Andrés & 645 & - & - & 105 & 16.28 & 540 & 83.72 \\
\hline Chocó & 983 & 86 & 8.75 & 13 & 1.32 & 884 & 89.93 \\
\hline Valle del Cauca & 703 & 434 & 61.74 & 184 & 68.40 & 85 & 12.09 \\
\hline Total & 5141 & 1294 & 25.17 & 603 & 11.73 & 3244 & 63.10 \\
\hline
\end{tabular}

grupo afrodescendiente. Por ejemplo en los departamentos de Chocó, Valle y Nariño se encuentra mayor población afro y también es más elevada la tasa, mientras que Putumayo tiene la cifra más baja en ambas variables. San Andrés, igual que la Guajira, tiene un alto componente de población afro, además tiene otro componente étnico de riesgo, que es la población inmigrante de origen mediterráneo y la población raizal, que también explican la alta tasa encontrada de hemoglobinas anormales (Tabla 6).

Otros estudios realizados para población colombiana, identifican las mismas variantes de hemoglobinas por departamento con cifras similares, a pesar de que el tamaño muestral y los criterios de muestreo son diferentes para cada estudio. El fenotipo anormal más común es el heterocigoto para hemoglobina S, excepto el estudio de Buenaventura que muestra el heterocigoto $C$ con más frecuencia que el heterocigoto $\mathrm{S}$, aunque éste también es alto. El total de hemoglobinopatías en este estudio fue de $7 \%$, resultado esperable considerando que se tomaron muestras de regiones de alto riesgo de hemoglobinopatías como San Andrés y de bajo riesgo como Putumayo, con componente étnico indígena alto, lo cual se vio reflejado en la correlación significativa encontrada entre proporción de población afro y proporción de hemoglobinopatías encontradas (Tabla 7).
Tabla 6. Distribución de hemoglobinas anormales encontradas, por ascendencia afro, según departamento.

\begin{tabular}{|l|c|c|c|c|}
\hline Departamento & $\begin{array}{c}\text { Hemoglobinas } \\
\text { anormales } \\
\mathbf{N}\end{array}$ & $\begin{array}{c}\text { Tasa } \\
\mathbf{\%}\end{array}$ & $\begin{array}{c}\text { Distribución de } \\
\text { hemoglobinopatias } \\
\text { por departamento } \\
\text { \% }\end{array}$ & $\begin{array}{c}\text { Autorreconocimiento } \\
\text { como } \\
\text { Afrodescendiente (7) } \\
\%\end{array}$ \\
\hline Putumayo & 5 & 1.5 & 2.2 & 5.47 \\
\hline Nariño & 53 & 7.1 & 23.2 & 18.8 \\
\hline Guajira & 27 & 4.1 & 11.8 & 14.82 \\
\hline San Andrés & 49 & 9.1 & 21.5 & 56.98 \\
\hline Chocó & 75 & 8.5 & 32.9 & 82.12 \\
\hline Valle del Cauca & 19 & 22.4 & 8.3 & 27.2 \\
\hline Total & $\mathbf{2 2 8}$ & $\mathbf{7 . 0 2}$ & $\mathbf{1 0 0 . 0}$ & - \\
\hline
\end{tabular}

\section{Conclusiones}

La tasa de hemoglobinas anormales según este estudio, es elevada, toda vez que alcanza $7 \%$ de todos los recién nacidos, con variaciones por región según su composición étnica. Esto plantea un riesgo para tener casos homocigotos, los cuales aumentan la mortalidad perinatal, así como los heterocigotos aumentan las tasas de morbilidad por

Tabla 7. Fenotipos de hemoglobinas anormales encontrados según diferentes estudios en muestras colombianas.

\begin{tabular}{|c|c|c|c|c|c|c|c|}
\hline \multirow[t]{2}{*}{ Estudio } & \multirow{2}{*}{$\begin{array}{c}\text { Muestra } \\
\text { N }\end{array}$} & \multicolumn{6}{|c|}{ Fenotipo en \% } \\
\hline & & F/AS & $\mathbf{F} / \mathbf{S}$ & F/AC & F/SC & otro & total \\
\hline Fonseca et al. (11) (Altiplano cundiboyacense) & 300 & - & - & 0.3 & - & 0.3 & 0.6 \\
\hline Bernal MP et al. (12) (Islas de San Andrés y Providencia) & 544 & 6.8 & 0.2 & 2.9 & 0.2 & 4.3 & 14.3 \\
\hline Villarreal et al. (13) (San Andrés) & 151 & 7 & - & - & - & - & 7 \\
\hline Alvear et al. (14) (Cartagena) & 1755 & 4.5 & - & 1 & 0.06 & - & 5.6 \\
\hline Satizábal et al. (15) (Cali) & 1000 & 5.12 & - & 2.19 & 0.10 & 0.62 & 8.1 \\
\hline Restrepo et al. (16) (Medellín) & 2627 & 2.01 & - & 1.44 & - & 0.03 & 3.53 \\
\hline Bernal M et al. (17) (Buenaventura) & 399 & 4.8 & - & 5.8 & 0.3 & 0.8 & 11.5 \\
\hline Este estudio en seis departamentos & 3244 & 4.65 & 0.12 & 1.88 & 0.06 & 0.3 & 7.01 \\
\hline
\end{tabular}


problemas respiratorios, musculoesqueléticos, digestivos, cardiovasculares y otros asociados a las hemoglobinopatías.

Para reducir la carga en salud aportada por las hemoglobinopatías, es necesario seguir las recomendaciones de la Organización Mundial de la Salud, en el sentido de hacer el tamizaje neonatal para las hemoglobinas anormales, considerando su fácil detección, el bajo costo y la posibilidad de iniciar manejo clínico para reducir la morbilidad. Cuando el tamizaje no sea posible, entonces en el control de crecimiento y desarrollo, está la oportunidad de captar casos mediante la clínica y los exámenes de sangre rutinarios, pero es necesario mejorar la capacidad diagnóstica del laboratorio y sospechar la enfermedad en las poblaciones afrocolombianas.

$\mathrm{Si}$ bien este estudio presenta sesgos propios de una muestra por conveniencia, de tamaño reducido y orientada a encontrar casos, la conclusión es que es posible utilizar las muestras de sangre seca en papel whattman 903, actualmente en uso para el tamizaje de hipotiroidismo congénito, con fines de implementar el tamizaje ampliado a las hemoglobinopatías. La Ley 1392 de 2006 de la República de Colombia, le protege el derecho a los niños y niñas para que sus problemas de salud relacionados con las anomalías congénitas sean diagnosticados, tratados y prevenidos en forma oportuna. Lo que sigue es estudiar las otras regiones del país, con composición étnica diferente, pero con riesgo dado que todo el país tiene amplio mestizaje y seguir promoviendo que se haga el tamizaje ampliado a éstas y a otras enfermedades. En el contexto latinoamericano, ya hay varios países que tienen implementado el tamizaje mediante el análisis de masas en tándem, que permite identificar el rasgo falciforme además de otras enfermedades metabólicas, siendo una eficaz herramienta para reducir la mortalidad infantil.

\section{Fuentes de financiación}

Financiado en su totalidad por el Instituto Nacional de Salud.

\section{Declaración de fuentes de financiación y posibles conflictos de interés}

La investigación que dio origen a este manuscrito, hace parte de las actividades misionales del Instituto Nacional de Salud, en cuanto a estudiar problemas de salud pública, como es el caso de las hemoglobinopatías, en razón a ello, la financiación en su totalidad fue por parte del Instituto Nacional de Salud, Subdirección de Red Nacional de Laboratorios. Las muestras para análisis fueron enviadas al Instituto Nacional de Salud por cuenta de los Laboratorios Departamentales de Salud Pública participantes.

\section{Agradecimientos}

Nuestro agradecimiento a los Laboratorios Departamentales de Salud Pública de Nariño, Putumayo, Valle del Cauca, Chocó, la Guajira y San Andrés Islas.

\section{Referencias}

1. McKenzie SB. Hematología clínica. 2a. ed. Bogotá D.C. Manual moderno. 2000 p.179-213.

2. Turgeon ML. Hematología clínica. Teoría y procedimientos. 1a. ed. Bogotá D.C. Manual moderno. 2006. p.179-99.

3. ACOG Practice Bulletin. Clinical Management Guidelines for ObstetricianGynecologist. Obstet Gynecol 2007; 109: 229-37.

4. Calvo JM, Zapata MF, Cuesta J, De la Iglesia I, Ropero P, Carreter E, Sicilia F. Prevalencia de hemoglobinopatías en mujeres gestantes en el área sanitaria de Lanzarote. An Med Interna 2006; 23(5): 206-12.

5. Modell B, Darlison Matthew. Global epidemiology of haemoglobin disorders and derived service indicators. Bulletin of the World Health Organization 2008 ; 86: $480-7$

6. Abarca G, Navarrete M, Trejos R, de Céspedes C, Saborío M. Hemoglobinas anormales en la población neonatal de Costa Rica. Biol Trop 2008; 56(3): 995 1001

7. Colombia. Departamento Administrativo Nacional de Estadística. Dirección de censos y demografía. Colombia una nación multicultural: su diversidad étnica. Bogotá, D.C. 2007. [consultado el 5 de octubre de 2011]. disponible en http:// www.dane.gov.co/files/censo2005/etnia/sys/colombia_nacion.pdf

8. Hocking DR. The separation and identification of hemoglobin variants by isoelectric focusing electrophoresis: An interpretive guide. PerkinElmer Life Sciences Cod Number HC-60. Akron OH, USA.1996.

9. Mantikou E, Harteveld CL, Giordano PC. Newborn screening for hemoglobinopathies using capillary electrophoresis technology: Testing the Capillarys ${ }^{\circledR}$ Neonat Fast Hb device. Clinical Biochemistry. 2010; 43(16-17): 1345-50.

10. Ji-Eun K, Bo-Ram K, Kwang-Sook W, Jeong-Man K, Joo-In P, Jin-Yeong H Comparison of capillary electrophoresis with cellulose acetate electrophoresis for the screening of hemoglobinopathies. Korean J Lab Med 2011; 31(4): 238-43.

11. Fonseca D, Angel C, Bernal P, Bustos I. Estimación de la frecuencia de hemoglobinopatías en el altiplano cundiboyacense. Biomédica 1994; 14(Sup.1): 12.

12. Bernal M. del P, Giraldo A, Bermudez AJ, Moreno E. Estudio de la frecuencia de hemoglobinopatías en las islas de San Andrés y Providencia. Biomédica 1995; 15: 5-9.

13. Villarreal E, Bermúdez AJ. Tamizaje para hemoglobinopatías. Inf Quinc Epidemiol Nac 2002; 7(15): 248-250.

14. Alvear C, Barboza M, Alayón AN, Viola M, Araque LM. Detección precoz de hemoglobinopatías en recién nacidos de la clínica maternidad Rafael Calvo de Cartagena: estudio piloto 2010. [En línea]. Memorias taller anual TSH Neonatal 2010. [consultado el 25 noviembre de 2011]. Disponible en: http://www.ins.gov. co/?idcategoria $=92469$

15.Satizábal JM, Neuta PA, Solorzáno M, Torres J, Somovar P, et al. Hemoglobinopatías en el Suroccidente Colombiano. [En línea]. Memorias taller anual TSH Neonatal 2010. [consultado el 25 noviembre de 2011]. Disponible en: http://www. ins.gov.co/?idcategoria $=92468 \#$

16. Restrepo F, Loaiza N, Arrubla M, Cossio S, Ordoñez J. Estudio de la prevalencia de anemia falciforme y otras hemoglobinopatías en población de recién nacidos del área metropolitana de Medellín. [En línea]. Memorias taller anual TSH Neonatal 2010. [consultado el 25 noviembre de 2011]. Disponible en: http://www.ins.gov. co/?idcategoria $=82033 \#$

17. Bernal M, Collazos A, Bonilla RD, Tascón EP. Determination of the prevalence of hemoglobin S, C, D, and G in neonates from Buenaventura. Colomb Med 2010 41: $141-7$ 